\title{
Placement of a single anterior odontoid screw for type II odontoid fractures in a setting with limited resources
}

\author{
Ahmed Hamdy Ashry ${ }^{1}$ and Hashem Mohamed Aboul-Ela ${ }^{1,2^{*}}$ (D)
}

\begin{abstract}
Background: Treatment of odontoid fractures with odontoid screws is an established method for fusion. However, it is facilitated by using advanced equipment in the operating theatre not always available as in developing countries.

Objectives: In this study we aim to outline the important key points for successful placement of odontoid screw in the absence of advanced tools, e.g. navigation or O-arm.

Materials and methods: Fifteen patients suffering from type II odontoid fractures were managed with single odontoid screw. Reduction of the fractures was achieved. A simple instrument set was used with the help of a single plane image intensifier. Longitudinal incision was used in 4 cases, and transverse incision was used in the remaining eleven cases. Anatomical identification of the midline helped to mark the entry point. The entry point used was millimetres below the anteroinferior edge of the axis vertebra. A short-headed screw was used.

Results: Road traffic accident was the only mechanism of trauma reported in our study. All of the patients were neurologically intact. All of the patients remained neurologically intact after the procedure. There were no instances of wrong trajectory of the screw in our study. During follow-up, we found good union of all the fractures. Only 2 cases suffered from mild dysphagia.

Conclusion: Fixation of odontoid fractures by anterior odontoid screws appears to be a safe and feasible procedure even in underequipped theatres. Anatomical knowledge helps in determining midline. Various modifications can help in overcoming shortcomings.
\end{abstract}

Keywords: Odontoid, Screw, Type II, Fracture

\section{Introduction}

Odontoid fractures account for $5-15 \%$ of all cervical spine injuries. Type II odontoid fractures are the most common axial fractures and may result in spinal cord injury due to atlantoaxial instability [1].

Treatment of such injuries is debatable. Rigid immobilization in brace or halo vest is impractical and carries the risk of high morbidity and failure rates. Posterior atlantoaxial fusion restricts the axial rotation at this joint. Anterior odontoid screw provides direct way of

\footnotetext{
* Correspondence: hashem.tawfik@kasralainy.edu.eg

'Department of Neurosurgery, Faculty of Medicine, Cairo University, Cairo,

Egypt

${ }^{2}$ Cairo, Egypt
}

Springer Open

(c) The Author(s). 2019 Open Access This article is distributed under the terms of the Creative Commons Attribution 4.0 International License (http://creativecommons.org/licenses/by/4.0/), which permits unrestricted use, distribution, and reproduction in any medium, provided you give appropriate credit to the original author(s) and the source, provide a link to the Creative Commons license, and indicate if changes were made. fusion with high rates of healing and motion preserving at C1-C2 joint [2].

Nowadays, odontoid screws can be used with high accuracy and less time consuming with the aid of recent advanced tools in neurosurgery like $\mathrm{O}$ arm and navigation $[3,4]$. In low-income countries like Egypt we lack the resources for these tools. In this study we discuss the important key points for successful placement of odontoid screw in the absence of these recent tools.

\section{Materials and methods}

Fifteen patients from a cohort group were included in our study. They were managed with anterior single odontoid screw placement for type II odontoid fractures due to road traffic accidents (RTA) from January 2016 to 
December 2018 in the Department of Neurosurgery, Kasr Alainy School of Medicine, Cairo University, Egypt. After arrival to the casualty department, $\mathrm{ABCD}$ protocol was applied. CT cervical spine was done to assess the type and shape of the fracture and exclude other fractures. CT scans were performed using the following machines: General Electric ${ }^{\circledR}$ Brightspeed (USA) and Siemens ${ }^{\circ}$ Somatom Emotion (Germany). MRI was used to assess disco-ligamentous structures especially transverse atlantal ligament (TAL) and to exclude spinal cord injury. MRI was performed using $1.5 \mathrm{~T}$ Siemens Magnetom Symphony Maestro Class, Syngo MR 2002B (Siemens Medical system Inc., Erlangen, Germany). The nature of the disease, its sequelae, plan of management with possible alternatives and complications of each were explained clearly to the patient and family members. Written consent for the management was obtained from all of the patients. We used locally manufactured cannulated and partially serrated lag screw with a short head. Postoperative CT was done to assess the trajectory of the screw and reduction of the fracture fragment. Dynamic x rays after 6 weeks were requested to assess any hardware failure or malunion.

All of the patients had recent odontoid fractures which coursed obliquely from the antero-superior to posteroinferior portion of the dens with intact TAL. This allowed perpendicular trajectory of the screw over the fracture line. Bone quality was also considered so all cases with different shapes of fracture, osteopenia, comminuted or pathological fractures were excluded from this management. All patients with displaced fracture were put in traction under fluoroscopy preoperatively to confirm reduction of the displaced fragment.

Preoperative examination the patient body habitus is very critical. All cases with short neck, barrel chest, and severe thoracic kyphosis were excluded. Abnormal body habitus can impede the right trajectory of the screw and increases the risk of its failure.
The following instruments were used (Fig. 1): 2 Cloward retractors, hand retractor, Kirschner wire (K-wire) of 1.2 $\mathrm{mm}$ diameter with sharp tip, cannulated drill bit of 3.2 $\mathrm{mm}$ external diameter and $1.2 \mathrm{~mm}$ internal diameter, hand drill, non-self-tapping partially threaded lag screw of $4 \mathrm{~mm}$ diameter with short head, and straight screwdriver.

The patient is placed in supine position with a pad under the interscapular region to allow neck extension. A piece of gauze is used to keep the patient's mouth open to obtain transoral views instead of the unavailable translucent mouth gag. C-arm fluoroscopy is used to check the reduction of the fractured fragment before starting the procedure. The fractured segment can be classified into 3 types: anteriorly displaced, neutral and posteriorly displaced. The head is placed in extension, with the aid of cervical traction if needed, to reduce anteriorly displaced fractures. It is required for facilitating the trajectory of the screw in all fracture types. In posteriorly displaced fractures, head is placed in the neutral position or mild extension to open a corridor for reaching the exact entry point at the inferior surface of C2. During introduction of the k-wire, head manipulation by pressure on the chin (military chin-tuck position) helps in bringing the fracture line perpendicular to the wire and thus achieving good trajectory for a good reduction.

In most cases we use a horizontal skin incision in the skin crease from the medial border of sternomastoid muscle to the midline based on C5-6 level on the right side. In 4 cases we used a vertical skin incision along the medial border of the sternomastoid muscle.

Blunt dissection is performed within the avascular plane to reach the pretracheal fascia and extended rostrally to C2-3 space which is confirmed by $\mathrm{C}$-arm fluoroscopy. Due to the unavailable special retractor system, with acutely angled superior retractor, we used two selfretaining Cloward retractors which helped the exposure

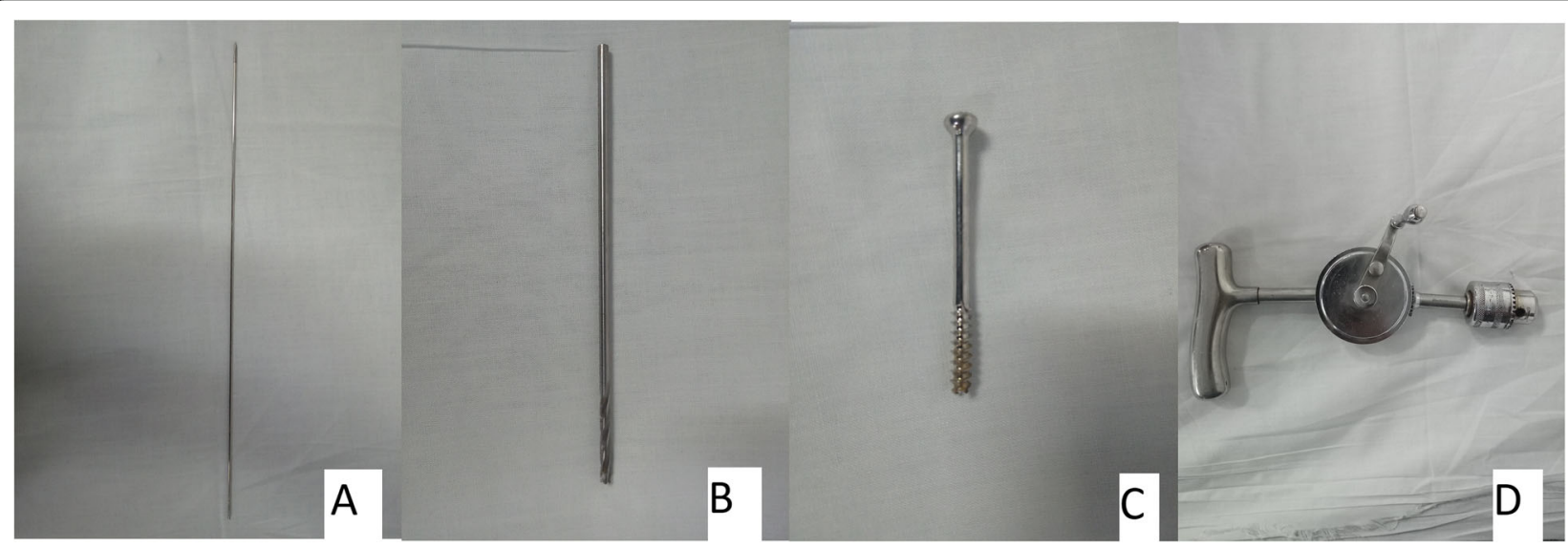

Fig. 1 Instruments set used in our series. a K-wire of $1.2 \mathrm{~mm}$ diameter with sharp tip. b Cannulated drill bit of $3.2 \mathrm{~mm}$ external diameter and 1.2 $\mathrm{mm}$ internal diameter. c Non-self-tapping partially threaded lag screw of $4 \mathrm{~mm}$ diameter with short head. $\mathbf{d}$ Hand drill 
of a working channel in the vertical and horizontal directions. Median part of $\mathrm{C} 2-3$ disc was removed to access the anteroinferior surface of $\mathrm{C} 2$ body.

We made sure that the patient's head is in the midline position. The midline is identified anatomically by palpation of the midline keel and dividing the distance equally between both longus colli muscles. With the aid of Carm fluoroscopy in the A-P view the middle point of the anteroinferior edge of $\mathrm{C} 2$ body is confirmed. We placed the sharp-tipped K-wire a few millimetres deep to this point and we changed the $\mathrm{C}$-arm to lateral views to confirm the trajectory before penetration (Fig. 2). During introduction of the K-wire we did alternating AP and lateral views by the $\mathrm{C}$-arm to ensure right trajectory. Hollow tools and hollow screws are inserted into the bone over the K-wires and are advanced to the tip of the dens. We tightened the lag screw till being impacted into the inferior endplate of $\mathrm{C} 2$ body (owing to its short head) with its anterior edge covering the head of the screw (Fig. 3).

\section{Results}

In our study there was male predominance (12 males, 3 females) with age ranged from 22 to 68 years. Road traffic accident was the only mechanism of trauma reported in our study. All of the patients were neurologically intact with no MRI documentation of spinal cord contusion.

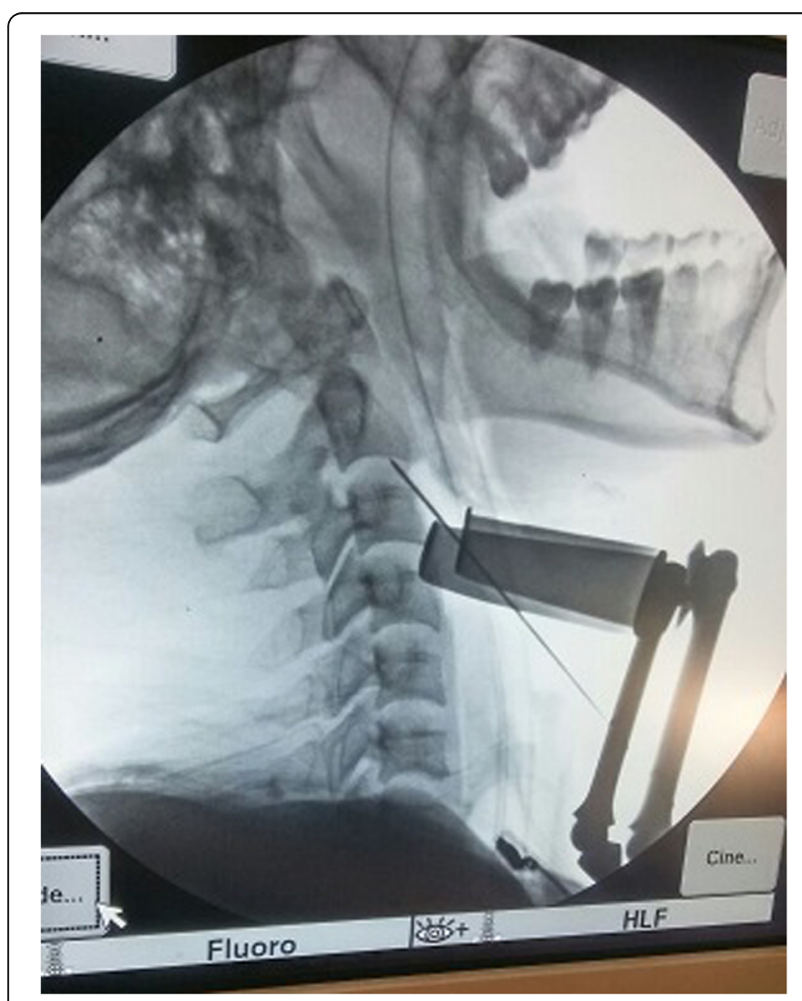

Fig. 2 Intraoperative image: the entry point is marked, and trajectory is achieved using the k-wire on lateral view
There were other injuries associated with the condition in 5 patients. All of the patients remained neurologically intact after the procedure (Table 1). There were no instances of wrong trajectory of the screw in our study. During the follow-up visits, we found good union of all the fractures without any reports of screw malfunctioning. Only 2 cases suffered from mild dysphagia which disappeared few days after the operation. There were no wound related complications. There was no mortality reported in our study.

\section{Discussion}

Management of odontoid fractures by anterior odontoid screw may pose a challenge especially in an underequipped operating theatre. We reviewed the available literature for the management of odontoid fractures by odontoid screws. We tried to underline the main cornerstones for the success of this procedure. These fall into: selection of the patient with proper planning, proper reduction of the fracture and achieving a good trajectory for the placement of the screw. We tried to adopt all the mentioned tips and tricks as much as we could, or we made some modifications to overcome shortcomings due to the underequipped operating theatre, being in a developing country.

Anterior odontoid screw should be performed with fractures extending from the anterosuperior part to the posterosuperior part. In this fracture type the screw can be placed perpendicular to the fracture line. This is classified Grauer type IIb [5]. All of our patients had this type of fracture. Other fracture types in which the line extends obliquely from the posterosuperior to the anterosuperior edge is not ideal for odontoid screw because reduction of the fractured segments cannot take place.

Good bone quality is vital for healing of fractures. Odontoid fractures are liable to poor healing due to lack of trabeculae at the cortical bone in the base of odontoid. Patients with osteoporosis comminuted fractures or pathological fractures are not candidates for surgery. Body shape may pose additional challenges to the operation (e.g. barrel chest, short neck, subaxial cervical canal stenosis, or severe thoracic kyphosis). It may be difficult for drilling and screw placement in these cases [1].

In our study we used a single screw in all patients. Placement of 2 screws yields a higher fusion rate in patients older than 70 years [6]. However, no difference in outcome or stability in the rest of patients was found $[7,8]$. In our situation, placing two screws is not feasible due to economic reasons. Moreover, placement of 2 screws may be more technically demanding [1].

The main tools we lack in our theatre were the O-arm or navigation, an angled retractor and specially designed instruments for this procedure. Here we describe how we overcame these problems. The usually used incision is made at the level of C5-6, which allows easier 


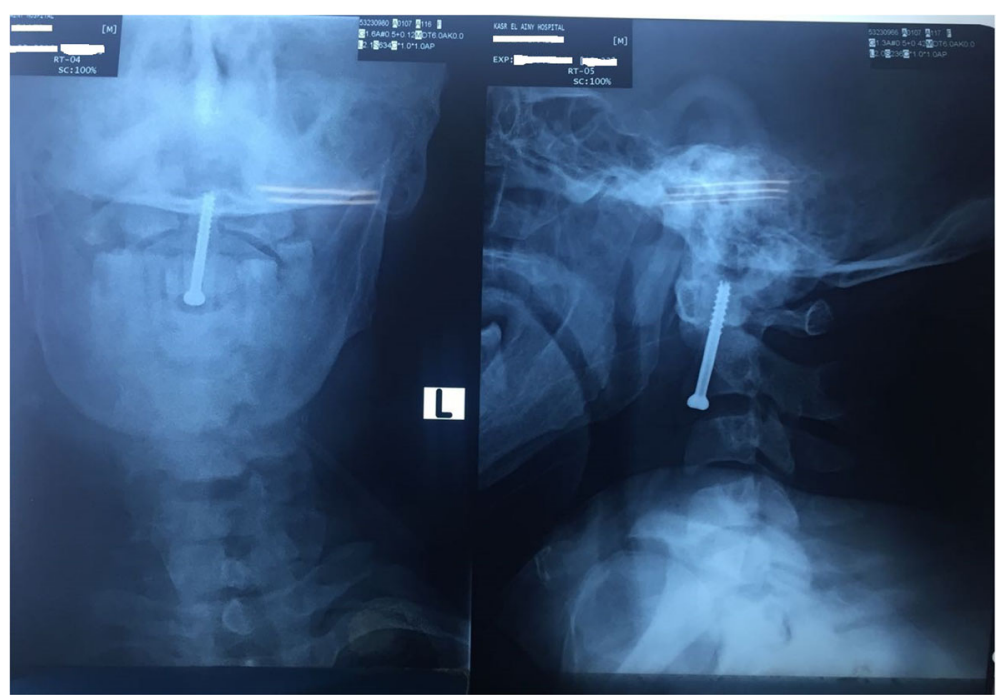

Fig. 3 Postoperative images showing the final position of the screw. The screw is impacted into the inferior endplate of C2 body (owing to its short head) with its anterior edge covering the head of the screw

placement of the screw due to the more feasible trajectory. Due to the unavailability of a special angled retractor as previously described [7], we used the basic retractor system present in our theatre: Cloward retractor. At first, we used a hand retractor in the cranio-caudal plane. However, the assistant could not maintain the exact retraction and was rather tiring due to the presence of abundant soft tissues in the cranial direction. Therefore, 2 Cloward retractors are used to help in both the horizontal and vertical planes. Campos and colleagues described an approach with a higher incision opposite C2-3 in contrast the usual opposite C5-6. They claimed that this approach avoided excessive tissue damage by retraction or dissection and blind entry of the instruments. However, we found the trajectory through the high incision somewhat problematic with our non-angled instrument set [9]. In spite of being less cosmetically accepted, we found that using a longitudinal incision had the advantages of both low and high incisions. The need for rostral traction to reach C2-3 disc is avoided as in the high incision and a good trajectory is achieved as in the low incision.

Anatomical identification of the midline is crucial in the determination of the entry point. This was done after equally dividing the distance between the two longus

Table 1 Summary of results

\begin{tabular}{|c|c|c|c|c|c|c|}
\hline & Age & Sex & Fracture Displacement & Incision & Associated injury & Postoperative complications \\
\hline 1 & 34 & M & Anterior & Transverse & & \\
\hline 2 & 45 & M & Anterior & Transverse & Fissure skull fracture & Dysphagia \\
\hline 3 & 22 & M & Anterior & Transverse & & \\
\hline 4 & 61 & F & Posterior & Longitudinal & & \\
\hline 5 & 54 & M & Neutral & Transverse & & \\
\hline 6 & 28 & M & Anterior & Transverse & Fracture clavicle & \\
\hline 7 & 39 & $\mathrm{~F}$ & Posterior & Transverse & & \\
\hline 8 & 41 & M & Anterior & Longitudinal & & \\
\hline 9 & 28 & M & Anterior & Transverse & Compound fracture tibia & \\
\hline 10 & 56 & F & Posterior & Transverse & & \\
\hline 11 & 68 & M & Anterior & Transverse & & Dysphagia \\
\hline 12 & 27 & M & Neutral & Longitudinal & Simple depressed skull fracture & \\
\hline 13 & 38 & M & Anterior & Transverse & & Dysphagia \\
\hline 14 & 47 & M & Posterior & Transverse & Fracture rib & \\
\hline 15 & 51 & M & Neutral & Longitudinal & & \\
\hline
\end{tabular}


colli muscles on either side of the body of $\mathrm{C} 2$. An important anatomical landmark seldom mentioned in literature, but useful in our series is the palpable midline keel in the midpoint of the anterior surface of dens [10]. Identification of the midline was confirmed with the help of fluoroscopy. The main use of the $\mathrm{A} / \mathrm{P}$ view is the identification of the midline. During introduction of the $\mathrm{K}$-wire we did alternating $\mathrm{A} / \mathrm{P}$ and lateral views by the single plane image intensifier to ensure right trajectory (due to the unavailability of biplanar o-arm or 2 image intensifiers). The major disadvantage of this method we found was the prolonged fluoroscopy time, thus requiring more operative time, radiation exposure and patience. The repeated switching between $\mathrm{A} / \mathrm{P}$ and lateral views may degrade the quality of $\mathrm{A} / \mathrm{P}$ images and may also be a risk factor for surgical site infection [10]. Therefore, proper knowledge of the anatomical landmarks may decrease the need for the use of fluoroscopy during the procedure and being dependent on it.

Drilling was done by using a hand-drill instead of the unavailable power drill. Hand drill allows for better control than a powered drill due to direct feedback between the user's input and the sensation of the drill bit turning. During the procedure one can have intraosseous feeling then some release at the fracture line then intraosseous feeling again when the fracture segment is entered. We found that it is safer especially in the absence of a tube system which protects the adjacent tissues from the instruments rotating at high speed. However, drilling with a hand-drill remains more time consuming. Pressure on the $\mathrm{k}$-wire may lead to breakage especially in the presence of metal fatigue, so we opted to use new never-used k-wires with sharp tip. This was to avoid the rare complication of $\mathrm{k}$-wire breakage during the procedure [11].

The entry point of the screw is poorly described in literature. However, the ideal entry point mentioned is the middle part of the anteroinferior angle of $\mathrm{C} 2$ body [7]. Lobo and colleagues tried different entry points in their series by either going millimetres above, millimetres below or exactly at the anteroinferior angle. They noted that for best fusion the screw must reach the apex of dens as it has the cortical highest bone density. They found out that placing the entry point above or anterior to the body had acceptable union rates [12]. However, this anterior position was linked in literature to the rare incidence of pharyngeal extrusion of the screw [13]. We prefer in our series to use of a short-headed screw. The entry point is a few millimetres deeper in the inferior surface of $\mathrm{C} 2$ to avoid breakdown of the body anterior to the screw trajectory. We aim at advancing the screw till it is impacted under the cover of the inferior endplate of $\mathrm{C} 2$. We found this step very useful in avoiding postoperative dysphagia which is not uncommon after this procedure and avoids screw pullout. This technique in fact is similar in its idea to the use of Herbert screw as described by Lee and colleagues (double threaded screw with no head) which is also buried beneath the edge of $\mathrm{C} 2$ and offers the same advantages [14]. Going deeper in the inferior surface of $\mathrm{C} 2$ and following the exact trajectory towards the tip of dens maybe hindered by the anterosuperior portion of $\mathrm{c} 3$ which may need trimming. Dysphagia usually occurs in the early postoperative period and decreases gradually. It may be related to retraction of pharynx and esophagus or dissection of soft tissues [9]. We tried to ameliorate this problem by loosening the retractor after introducing the k-wire. Another cause for dysphagia is the close relationship of the esophagus and pharynx to the anterior odontoid screw. This may even cause their perforation, which could be fatal if not treated carefully with help from other specialties [13].

\section{Conclusions}

Management of odontoid fractures by anterior odontoid screws may be problematic in the setting of an underequipped operating theatre. Modifications can be performed to achieve acceptable results. Proper selection of patients candidate for the procedure is crucial. A single screw can be used with favourable results. The absence of biplanar image intensifier and navigation can be overcome using alternating views of a single-plane image intensifier, but the prolonged fluoroscopy time may be a drawback. Sound knowledge of the anatomical landmarks may decrease the need for fluoroscopy. Using a longitudinal incision obviates the need for a special angled retractor and allows the use of a single Cloward retractor. A hand drill can be used instead of the pneumatic drill with similar results. We introduce a technical modification to the entry point of the screw. The screw is placed millimetres below the edge of the body to avoid breaking of the ventral segment. The use of a short-headed screw and lunging it into the lower endplate of dens to be buried helps in avoiding postoperative dysphagia either early or late (as in esophageal perforation).

In conclusion, fixation of odontoid fractures by anterior odontoid screws appears to be a safe and feasible procedure even in underequipped theatres.

\section{Acknowledgements Not Applicable. \\ Authors' contributions \\ HA and AA conceived of the study, and participated in its design and coordination and helped to draft the manuscript. AA performed data acquisition and its analysis. HA and AA reviewed the manuscript. All authors read and approved the final manuscript.}

\section{Funding}

This research received no specific grant from any funding agency in the public or commercial sector.

Availability of data and materials

The datasets used and/or analyzed during the current study are available from the corresponding author upon reasonable request. 


\section{Ethics approval and consent to participate}

This observational study was approved by the ethics committee of the Department of Neurosurgery, Kasr Alainy Faculty of Medicine, Cairo University on the 9th of December 2015. All participants provided informed written consent to participate in the study.

\section{Consent for publication}

Informed written consent to publish has been obtained from the participants to report individual patient data.

\section{Competing interests}

The authors declare that they have no competing interests.

Received: 6 February 2019 Accepted: 12 November 2019

Published online: 10 December 2019

\section{References}

1. Mazur MD, Mumert ML, Bisson EF, Schmidt MH. Avoiding pitfalls in anterior screw fixation for type II odontoid fractures. Neurosurg Focus. 2011;31(4):E7.

2. Smith HE, Vaccaro AR, Maltenfort M, Albert TJ, Hilibrand AS, Anderson $D G$, et al. Trends in surgical management for type II odontoid fracture: 20 years of experience at a regional spinal cord injury center. Orthopedics. 2008;31(7):650.

3. Rajasekaran $\mathrm{S}$, Jaiswal A, Shetty A. Role of intraoperative Iso-C based navigation in challenging spine trauma. Indian J Orthop. 2007:41(4):312.

4. Wu J-C, Wadhwa R, Than K, Mummaneni P. Complication avoidance and management using the $\mathrm{O}$-arm for odontoid screw fixation: technical note. Cureus. 2014;6(11):e225. https://doi.org/10.7759/cureus.225.

5. Grauer JN, Shafi B, Hilibrand AS, Harrop JS, Kwon BK, Beiner JM, et al. Proposal of a modified, treatment-oriented classification of odontoid fractures. Spine J. 2005:5(2):123-9.

6. Dailey AT, Hart D, Finn MA, Schmidt MH, Apfelbaum RI. Anterior fixation of odontoid fractures in an elderly population. J Neurosurg Spine. 2010;12(1):1-8.

7. Apfelbaum Rl, Lonser RR, Veres R, Casey A. Direct anterior screw fixation for recent and remote odontoid fractures. Neurosurg Focus. 2000;8(6):1-10.

8. Feng G, Wendlandt R, Spuck S, Schulz AP. One-screw fixation provides similar stability to that of two-screw fixation for type II dens fractures. Clin Orthop Relat Res. 2012;470(7):2021-8.

9. Campos WK. Anterior screw fixation for odontoid fracture using the direct approach at the C2-C3 level: case report and literature review. J Spine. 2013;3(2). https:/doi.org/10.4172/2165-7939.1000162

10. Bednar DA. Uncovertebral anatomic midline targeting for cervical disc arthroplasty. Global Spine J. 2012;2(1):47-50.

11. Sarkar C, Sharma MC, Deb P, Singh R, Santosh V, Shankar SK. Primary central nervous system lymphoma - a hospital based study of incidence and clinicopathological features from India (1980-2003). J Neurooncol. 2005; 71(2):199-204.

12. Lobo JPFM, Moutinho W, Serdoura AFM, Oliveira CF, Pinho AR. Anterior fixation of odontoid fractures: results. Rev Bras Ortop. 2017;53(5):532-6.

13. Lee EJ, Jang JW, Choi SH, Rhim SC. Delayed pharyngeal extrusion of an anterior odontoid screw. Korean J Spine. 2012;9(3):289.

14. Lee S-H, Sung J-K. Anterior odontoid fixation using a 4.5-mm Herbert screw: the first report of 20 consecutive cases with odontoid fracture. Surg Neurol. 2006 Oct;66(4):361-6.

\section{Publisher's Note}

Springer Nature remains neutral with regard to jurisdictional claims in published maps and institutional affiliations.

\section{Submit your manuscript to a SpringerOpen ${ }^{\circ}$ journal and benefit from:}

- Convenient online submission

- Rigorous peer review

- Open access: articles freely available online

- High visibility within the field

- Retaining the copyright to your article

Submit your next manuscript at $\boldsymbol{\nabla}$ springeropen.com 